\title{
The Smartphone as the Incumbent "Thing" among the Internet of Things
}

\begin{abstract}
The smartphone has been the ubiquitous computing platform in the past decade. However, emerging consumer Internet of Things (IoT) technology trends, such as smartwatches and smart speakers, promise the establishment of new ubiquitous platforms. We model two competing horizontally-differentiated platforms that each offer a smartphone and another smart device. This market diverges markedly from standard mixed bundling results when devices from the same vendor have super-additive utility. We show that the degree of a smart device's differentiation (relative to the smartphone) is the prime factor determining if it is profitable to deepen integration between a smart device with the incumbent smartphone platform. We provide managerial insights for technology strategy.
\end{abstract}

\section{Introduction}

Mobile computing in the form of smartphones is a dominant technological platform today [13]. However, the Internet of Things (IoT) promises a broad array of interconnected products ranging from tiny fitness trackers to smart homes.

The key question that this paper explores in a rigorous analytical-modeling approach is the following: How will the emerging trends of wearables (e.g., Apple Watch, Android Wear, Pebble) and smart-home appliances (e.g., Amazon Echo, Apple HomePod, Google Home) impact smartphones and the mobile computing platform that dominates devices today?

We focus on smartwatches and smart speakers as contrasting ends of the consumer IoT spectrum. At present, many vendors have developed smart devices that depend on an interoperable/compatible smartphone in order to function. Thus, the smart device functions as a "hardware app" that is a complement to its compatible smartphone platform and adds value to that platform

\footnotetext{
${ }^{1}$ A "single-homing" user - for example a consumer who purchase a smartwatch - will use only that one watch... at least until she replaces it with a newer model. A "multi-homing" user - for example a
}

through cross-sided network effects: The more apps and the more devices compatible to a given smartphone platform, the more valuable the platform itself, which attracts more users and attracts more apps (and potentially more compatible devices). In this scenario, an Apple Watch adds value to the iOS platform and attracts more users to it. In a competitive setting with single-homing users, ${ }^{1}$ the more "successful" the Apple Watch, the more the Apple ecosystems will benefit, and the Android ecosystem will suffer.

However, this situation is unlikely to be permanent. When a smart device becomes independent enough and developers build applications for them directly, the next-generation smart device may transform from a networked complement into a networked substitute.

We formalize the above observations in a competitive setting of two competing smartphone platforms [17], each offering complementary smart devices. We characterize market outcomes in terms of prices, market-shares, and profits and do sensitivity analysis examining how various market parameters affect the market outcomes. We identify various eras of connected device evolution and examine how nextgeneration connected devices in each era may affect today's dominant client-side computing platform, the smartphone.

\section{Background}

We discuss related literature on network effects in platforms and the Internet of Things.

\subsection{Network effects in platforms}

The paper is related to the broad network effects [10] and platform economics literatures [3,11,17]. This literature has been reviewed broadly by Rochet \& Tirole [18]. In addition, Heitkotter et al. [15] argued that twosided markets literature is useful in understanding the economics of mobile computing and related strategic

smartphone app developer - might decide to sell apps on both competing smartphone platforms, iOS and Android. 
question. In the rest, we highlight articles from the closest specific strand: bundling and tying in two-sided markets.

Research on information goods has shown that bundling might be an extremely profitable strategy [2, $14,16]$. Moreover, tying (when two or more products or services are offered only as a bundle and they are not available individually) can be used as a mechanism for foreclosing a market [5, 19]. Doganoglu \& Wright [9] analyzed the ability of an incumbent to use introductory offers to dominate a network market in the face of a more efficient rival. Amelio \& Jullien [1] showed that firms can use tying to subsidize participation in twosided markets, and that tying increases social welfare when network effects are strong. Choi [6] showed that tying induces more consumers to multihome and makes platform specific exclusive content available to more consumers, so tying can be welfare enhancing if multihoming is allowed. Gans [12] proposed antitrust remedies for tying and found conditions under which those remedies will be effective in improving social welfare. Choi \& Jeon [7] proposed a leverage theory of tying in two-sided markets, motivated by antitrust investigations concerning Google. Carlton \& Waldman [4] analyzed exclusive channel and revenue sharing strategies in a context of suppliers and retailers. Overall, most of this literature is concerned about welfare implications, and related antitrust issues, while our main focus is strategic interaction and the impact of technology.

\subsection{Internet of Things}

The Internet of Things theme has attracted substantial attention in industry reports and in the computer science and engineering literature [20].

In general, the concept refers to everyday things that have processing power, sensor, and actuator capabilities, and are interconnected with the rest of the Internet. As an example, as of September 2018, Apple announced the Series 4 of its Apple Watch with fitness tracking and other health-oriented capabilities. In the context of smart homes, Amazon announced a new series of smart speakers and related devices, including a smart microwave.

Over time, IoT will be ubiquitous, context-aware and provide ambient intelligence. Application areas could include supply chains, manufacturing, agriculture, energy, healthcare, etc. All these could be thought of as industrial IoT.

Our research is more close to the consumer IoT, which includes wearables, smart homes, smart cars and other related applications geared towards consumer markets. IoT promises the integration of physical and digital world and the creation of new value, but it also entails challenges such as security and privacy.

Overall, while the IoT is considered one of the most significant contemporary tech trends, there is limited economics research on the topic. Our research aims to start filling this gap.

\section{Model}

We consider a model in which two competing platform owners each offer a smartphone and a complementary product. We introduce our model with smartwatches as the complementary products, and then discuss smart speakers as a change in model parameters.

Each platform connects app developers with consumers to create positive cross-side network effects. Consumers have unit demand for at most one smartphone and at most one smartwatch (i.e., they single-home separately for each product), while developers can multi-home costlessly to make apps for any combination of the four products in a market. The network effects are independent for each product.

Consumers have horizontal preferences for products that we model as uniformly distributed locations in a Hotelling square such as Figure 1, with the $x$-axis related to smartwatches and $y$-axis related to smartphones, and misfit disutility arises from the distance "traveled" on each axis. Developers have design goals that are easier to realize on some products than others, so we distribute developers uniformly across a similar Hotelling square. We discuss correlated misfit costs in Subsection 4.4.

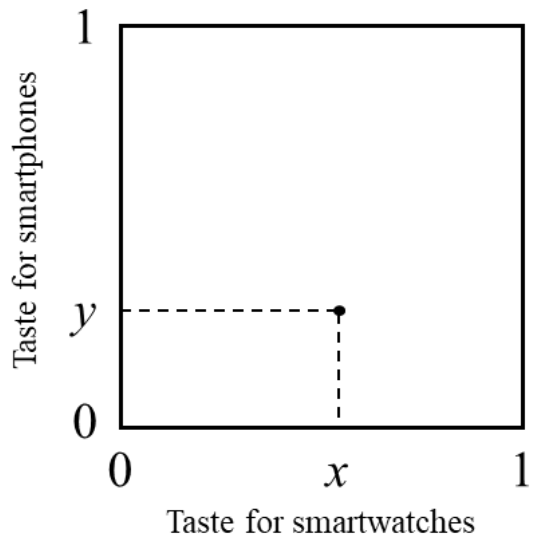

Figure 1: Hotelling square of preferences for smartphones and smartwatches.

Even with independent network effects and uncorrelated misfit costs, synergy exists between a vendor's products that increases the value to a developer or consumer. The exogenous parameter measuring this synergy $-s$ for developers and $S$ for consumers - is a strictly positive increase to value before price is 
considered. For example, a consumer purchasing both products from the same vendor receives $(1+S)$ times the sum of individual product utilities, less the disutility associated with prices.

We index a bundle by the watch and phone purchased (or developed for). $\varnothing$ indicates that the device is not part of the bundle, while $A$ and $B$ represent the vendors' offerings. Therefore, $\{A, A\}$ represents a bundle of the first vendor's smartwatch and smartphone, while $\{\varnothing, \mathrm{B}\}$ represents purchasing only the second vendor's smartphone.

In the following utility and value functions, $I$ is an indicator function; $C$ refers to consumer, $D$ to developer, $i$ to watch vendor, $j$ to phone vendor, $N$ to cross-side network effect, $P$ to price (or phone in subscripts), $Q$ to quantity/market-share, $S$ to synergy, $T$ to transport/ misfit cost, $U$ to consumer utility, $V$ to developer value, $\mathrm{W}$ in subscripts to watch, $x$ to smartwatch preference, and $y$ to smartphone preference. For parameters that affect Consumers and Developers separately, uppercase (e.g., $\left.N_{W}\right)$ applies to Consumers, and lowercase (e.g., $\left.n_{W}\right)$ applies to Developers.

$$
\begin{aligned}
& u_{W \emptyset}(x)=0 \\
& u_{W A}(x)=u_{W}+N_{W} q_{W A}-x \cdot T_{W} \\
& u_{W B}(x)=u_{W}+N_{W} q_{W B}-(1-x) T_{W} \\
& u_{P \emptyset}(y)=0 \\
& u_{P A}(y)=u_{P}+N_{P} q_{P A}-y \cdot T_{P} \\
& u_{P B}(y)=u_{P}+N_{P} q_{P B}-(1-y) T_{P} \\
& U_{i, j}(x, y)=\left[u_{W i}(x)+u_{P j}(y)\right] \times\left(1+I_{i=j} \cdot S\right)-P_{i, j} \\
& v_{W \emptyset}(x)=0 \\
& v_{W A}(x)=v_{W}+n_{W} Q_{W A}-x \cdot t_{W} \\
& v_{W B}(x)=v_{W}+n_{W} Q_{W B}-(1-x) t_{W} \\
& v_{P \emptyset}(y)=0 \\
& v_{P A}(y)=v_{P}+n_{P} Q_{P A}-y \cdot t_{P} \\
& v_{P B}(y)=v_{P}+n_{P} Q_{C(P B)}-(1-y) t_{P} \\
& V_{i, j}(x, y)=\left[v_{W i}(x)+v_{P j}(y)\right] \times\left(1+I_{i=j} \cdot s\right)-p_{i, j}
\end{aligned}
$$

All exogenous parameters are strictly positive except for taste indices, which range over the closed interval [0,1]. Market shares such as $Q_{W A}$ are endogenously determined and also range over $[0,1]$. We employ the common convention that cross-side network effects are proportional to market share on the other side of the network.

We introduce bundles in four eras. The first era represents the market before app-enabled smartwatches. The second era adds single-vendor bundles wherein a smartwatch only functions if the consumer also owns the matching smartphone. The third era improves smartwatches into stand-alone devices, which also permits consumers to mix and match between vendors if desired. Finally, the fourth era adds additional phonelike functions to smartwatches.

\section{Results}

We present the results of our model in chronological order across four eras. Formal analytical proofs of our Lemmas and Propositions are omitted due to space limitations in the proceedings. For the figures illustrating market outcomes, we choose model parameters that ensure that all intersections of interest occur within the Hotelling square.

\subsection{Smartphones only}

In the first era (before app-enabled smartwatches or smart speakers), the only bundles available to consumers are $\{\varnothing, \varnothing\},\{\varnothing, \mathrm{A}\}$ and $\{\varnothing, \mathrm{B}\}$.

With symmetric firms, the standard result obtains that each has a market share of one-half and charges a price equal to the transport cost $t_{P}$ (Figure 2). That is, firms compete away the network effect on the consumer side, which vastly increases the region of the parameter space that admits full coverage of Consumers.

Note that for symmetric firms, the standard result obtains that each has a market share of one-half and charges a price equal to the transport cost $t_{P}$. See the left panel of Figure 2. That is, firms compete away the network effect on the consumer side, which vastly increases the region of the parameter space that admits full coverage of Consumers.

Lemma 1: The condition for full market coverage of smartphone Consumers in the presence of network effects is $2 u_{P}+N_{P}\left(q_{P A}+q_{P B}\right) \geq 3 T_{P}$, which is a looser constraint than the standard $u_{P} \geq 3 T_{P}$ without network effects. Firms charge Consumers $T_{P}$ and Developers $v_{P} / 2+n_{P} / 4$ when an interior solution obtains, serving a fraction $\left[v_{P} / 2+n_{P} / 4\right] / t_{P}$ of Developers. Each firm's profit is $T_{P} / 2$ from Consumers plus $\left[\left(v_{P} / 2+n_{P} / 4\right)^{2}\right] / t_{P}$ from Developers.

The intuition behind this result is that network effects are competed away on the Consumer side with single-homing customers, but not on the multi-homing Developer side. The free utility from cross-side network effects makes full coverage on the Consumer side likely. Firms attract Developers independent of their activity with the competing firm, so the prices offered to Developers are those of local monopolists. 

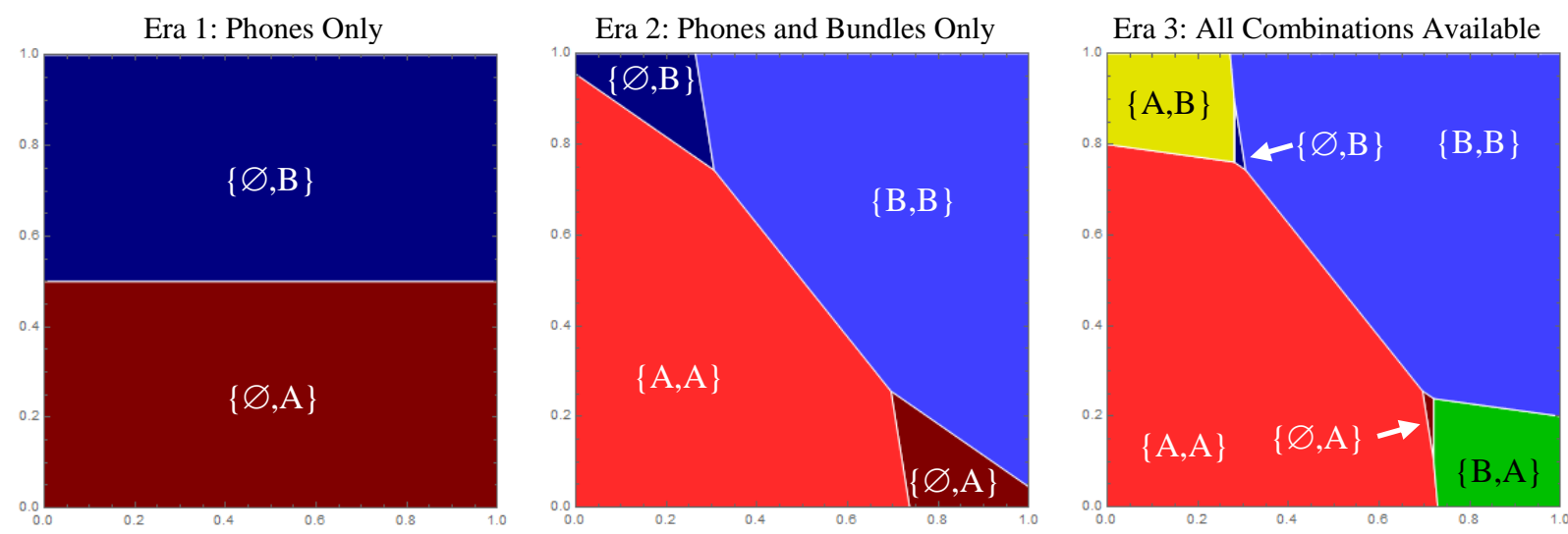

Figure 2. Consumer market shares across three eras with $S=1 / 4, T_{w}=5 / 8$ and $T_{P}=1 / 2$. The horizontal axis measures taste for watches while the vertical axis measures taste for phones. Developer market shares are much simpler because firms treat multi-homing developers as if the other firm does not exist.

We assume an interior solution for Developer market shares because this implies that some Developers sell on one platform but not the other, and this represents the real market for Developers. An interior solution places two mild constraints on transport/taste costs:

Assumption 1: Developer misfit costs are at least $t_{P}>\left(v_{P} / 2+n_{P} / 4\right)>0$..

Assumption 2: Consumer misfit costs are in the range $\left(2 / 3 \times\left[u_{P}+N_{P}\left(v_{P} / 2+n_{P} / 4\right) / t_{P}\right]\right) \geq T_{P}>0$.

\subsection{Smartwatches complement smartphones}

This era adds bundles $\{\mathrm{A}, \mathrm{A}\}$ and $\{\mathrm{B}, \mathrm{B}\}$ which have super-additive utility. Firms will keep the existing phone prices - which guarantee a fully covered
Consumer market for the older product - rather than bet on a risky new product being able to saturate the Consumer market with smartphone/smartwatch bundles. Given the more varied form factors and less flexible interface, it follows that misfit costs would be different in each dimension.

Assumption 3: Smartwatch tastes are stronger than smartphone tastes such that $T_{W}>T_{P}$ and $t_{W}>t_{P}$.

Proposition 1: Firms charge Consumers $T_{W}$ for smartwatches and Developers $v_{W} / 2+n_{W} / 4$ when an interior solution obtains, serving a fraction $\left[v_{W} / 2+n_{W} / 4\right] / t_{W}$ of Developers. Each firm's profit is $T_{W} / 2$ from Consumers plus $\left[\left(v_{W} / 2+n_{W} / 4\right)^{2}\right] / t_{W}$ from Developers.
Era 4: Future device with $\alpha=1 / 8$

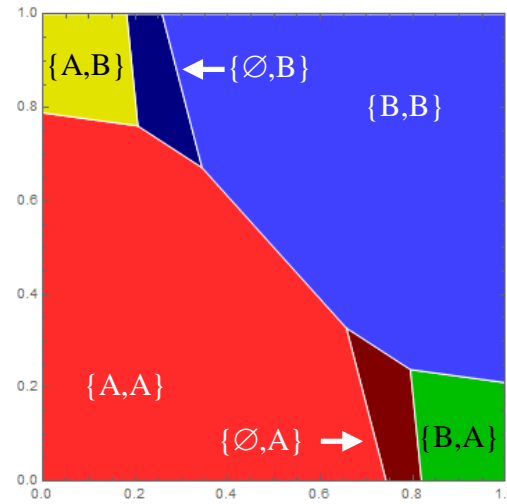

Era 4: Future device with $\alpha=1 / 4$

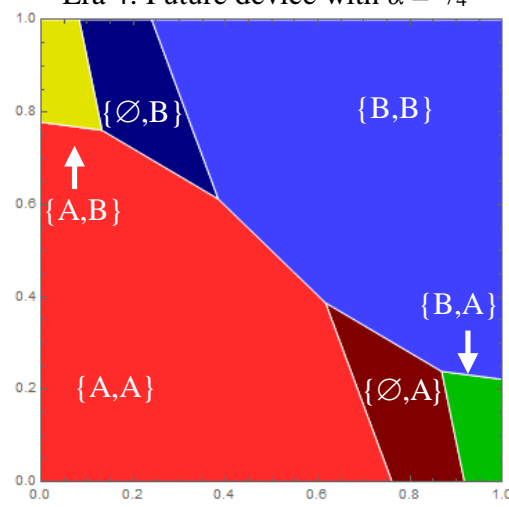

Era 4: Future device with $\alpha=3 / 8$

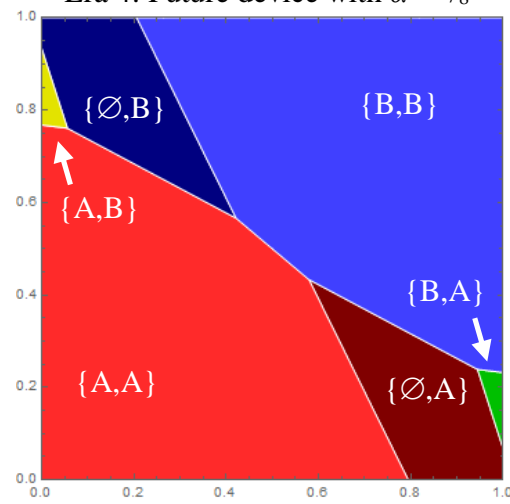

Figure 3. Consumer market shares with $S=1 / 4, T_{w}=5 / 8$ and $T_{P}=1 / 2$ when the future watch device becomes available over three values of $\alpha$. The horizontal axis measures taste for pure watches while the vertical axis measures taste for phones. 
The intuition behind this result is that the superadditive utility (i.e., complementarity) from a bundle induces Consumers with strong smartphone preferences or smartwatch preferences to purchase the bundle. If misfit costs are very low, all Consumers purchase bundles. Otherwise, some Consumers keep their original "bundles" of $\{\varnothing, \mathrm{A}\}$ and $\{\varnothing, \mathrm{B}\}$. Although the market shares have changed shape, indicating platform switching, each firm still sells smartphones to $50 \%$ of the Consumers and sells smartwatches to some fraction of its Consumers. We illustrate the impact in the middle panel of Figure 2. Smartwatches are revenue-enhancing for both firms.

\subsection{Stand-alone smartwatches}

This era adds bundles $\{A, \varnothing\},\{A, B\},\{B, \varnothing\}$ and $\{B, A\}$, for example enhancing the smartwatch with independent GPS and phone-call ability (e.g., Apple Watch Series 3). Era 3 allows most of the holdouts in Era 2 to purchase a smartwatch that meets their tastes, further improving vendor revenues once the market reaches equilibrium. The right panel in Figure 2 shows the market outcome.

Proposition 2: Adding unbundled smartwatches causes no Consumers to switch smartwatches, but some Consumers do purchase smartwatches who had not done so when they were only available in single-vendor bundles. Some of the Consumers who newly purchase smartwatches switch smartphones

The intuition behind this result is that anyone who purchased a smartwatch in Era 2 enjoys super-additive utility from a bundle, and a newly available unbundled smartwatch without super-additive utility will not cause any of those Consumers to switch. On the other hand, Consumers just below the top-left and just above the bottom-right were only induced to purchase those phones due to the synergy of bundling with their preferred smartwatch. For these marginal phone purchasers, the ability to pick one from $\mathrm{A}$ and one from $\mathrm{B}$ allows them to switch back to their preferred phone.

\subsection{Phone-like smartwatches}

In this era, a future watch with smartphone functionality becomes its own device with a blending of the transport costs. Note that the ability to place phone calls is not in itself a differentiating feature (or even unique to Era 4... it became available in Era 3). Rather, we refer to the smartwatch acquiring some of the look and feel of the firm's smartphone offering. For a given fraction $\alpha$ of "phone-ness," the utility function for firm A's future device is:

$$
\begin{aligned}
& u_{W A}(x, y)= \\
& u_{W}+N_{W} q_{W A}-\sqrt{1-\alpha^{2}} \cdot x \cdot T_{W}-\alpha \cdot y \cdot T_{P}
\end{aligned}
$$

Note that setting $\alpha=0$ recreates Era 3, but here we shall only consider the case of strictly positive $\alpha$.

Proposition 3: Adding smartphone features to the smartwatch, such that it causes a blend of misfit costs across the phone and watch dimensions, decreases smartwatch sales and firm profit.

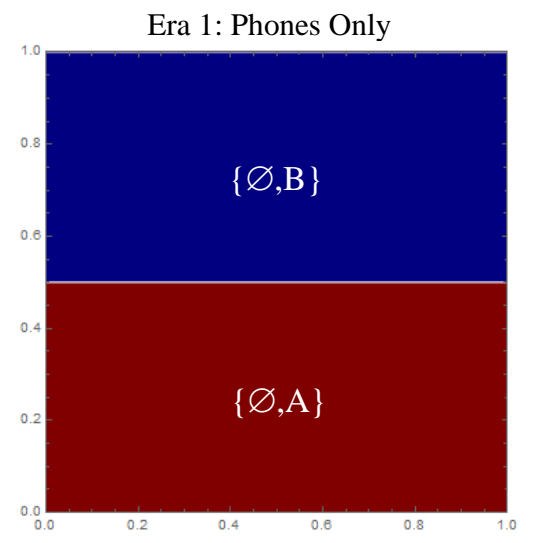

Era 2: Phones and Bundles Only

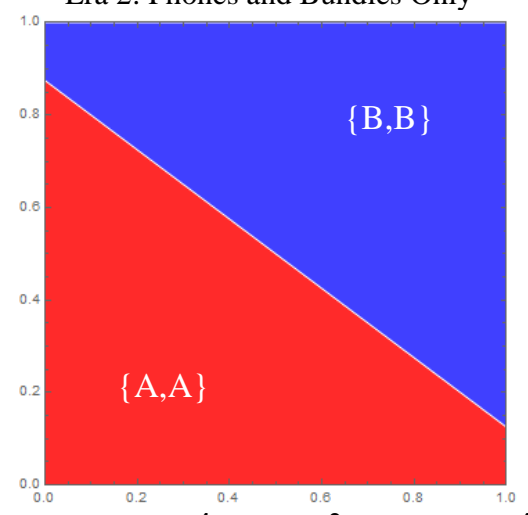

Era 3: All Combinations Available

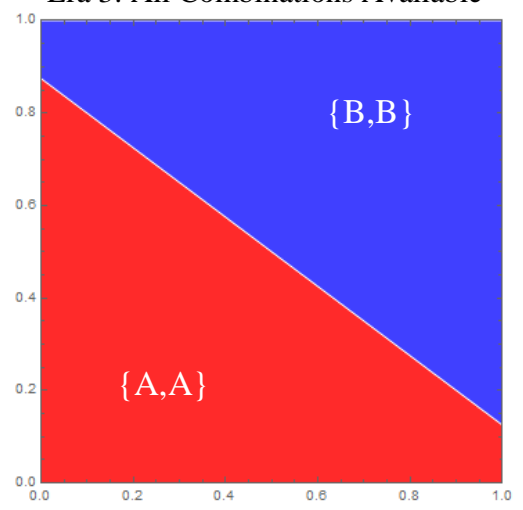

Figure 4. Consumer market shares with $S=1 / 4, T_{S}=3 / 8$ and $T_{P}=1 / 2$. The horizontal axis measures taste for speakers while the vertical axis measures taste for phones. 
Era 2: Phones and bundles only

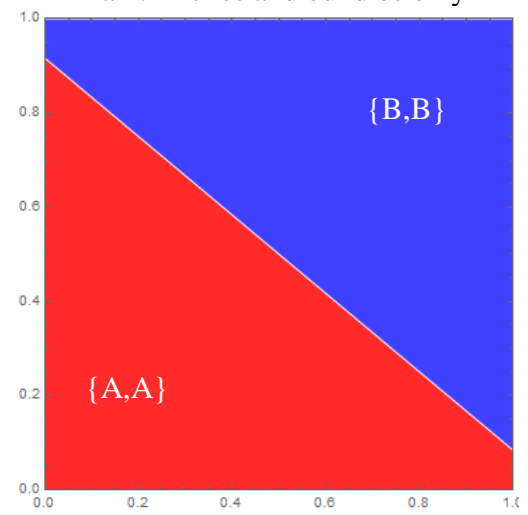

Figure 5. Consumer market shares with $S=1 / 4, T_{S}=5 / 8$ and $T_{P}=3 / 4$ (violating Assumption 2 as in Proposition 5). The horizontal axis measures taste speakers while the vertical axis measures taste for phones.
The intuition behind this result is that the indifference lines bordering $\{\varnothing, \mathrm{A}\}$ and $\{\varnothing, \mathrm{B}\}$ expand into the neighboring regions as $\alpha$ increases because (1) the mixed-vendor bundles become less compelling because the watches gain traits from the disfavored phone and (2) marginal single-vendor bundle Consumers suffer a bigger increase in phone-dimension misfit than their decrease in watch-dimension misfit.

A phone-like watch turns out to be a bad deal for vendors: The Consumers who mix a smartphone and a smartwatch from different vendors do so because they dislike the phone associated with their preferred watch. Making the watch more like that phone is a case where adding features actually hurts sales, as shown in Figure 3 wherein the phone-only region grows with higher $\alpha$.

\subsection{Smart speakers}

We consider the market for the less-mobile complement (smart home appliance, a.k.a. smart speaker) to be separate from that of the more-mobile complement (smartwatch) because the two are not substitutes for one another. The market for smart speakers is expected to evolve through the same four eras discussed above for smartphones. The difference is that as essentially immobile devices controlled primarily through voice commands, horizontal differentiation should be weaker than that of smartphones rather than stronger. The analysis would also apply to smart security systems, climate controllers, microwave ovens, etc.

Assumption 4: Smart speaker tastes are weaker than smartphone tastes such that $T_{S}<T_{P}$ and $t_{S}<t_{P}$.

Lemma 2: A complementary product with lower misfit costs than the smartphone's will always cover all
Consumers, and the market will exhibit less mixedproduct bundling that the smartwatch market.

The intuition behind this result is that since optimal prices can cover the Consumer market with smartphonestrength tastes, optimal prices will also cover the Consumer market for smart speakers once the market reaches equilibrium. Consumers also find their lesspreferred smart speaker less distasteful than their lesspreferred smartwatch, so single-vendor bundles are more compelling. See Figure 4 which has the same parameter values as in Figure 2 except with $T_{S}=3 / 8$.

Interestingly, while the introduction of $\alpha$ in Era 4 cannot possibly increase adoption, it may still be helpful to firms. The utility function for firm A's new device is:

$$
u_{S A}(x, y)=u_{S}+N_{S} q_{S A}-\sqrt{1-\alpha^{2}} \cdot x \cdot T_{S}-\alpha \cdot y \cdot T_{P}
$$

Proposition 4: Adding smartphone features to the smart speaker, such that it causes a blend of misfit costs across the phone and speaker dimensions, weakly decreases mixed-product bundling.

The intuition behind this result is that the smart speaker Consumer market is fully covered under weaker conditions than Assumption 2, so Consumers are more properly thought of as considering a phone to go with their speaker. The more phone-like the speaker becomes, the more the market shares resemble the phone-only market shares in Era 1, which increases the number of Consumers using their preferred smartphone.

However, there is a further consequence of the phone being a complement to the speaker. Consider a market in which firms focus on core customers' tastes [8] to the extent that the transport/misfit cost increases beyond the limit in Assumption 2. 
Proposition 5: Maintaining Assumptions 1, 3 and 4 but violating Assumption 2 leads to a market where some Consumers purchase a smartphone solely due to its supper-additive utility with a smart speaker. Consumers who purchase a single device purchase a smart speaker and no smartphone.

The intuition behind this result is that a group of Consumers with intermediate $y$ indices would prefer an outside option to the smartphones offered. This outside option would be the modern version of a feature phone (including some social media features, but no app ecosystem). Figure 5 shows that depending on model parameters, some of these Consumers may purchase a single-vendor bundle $\{\mathrm{A}, \mathrm{A}\}$ or $\{\mathrm{B}, \mathrm{B}\}$, but others may maximize their utility with the bundles $\{A, \varnothing\}$ and $\{B, \varnothing\}$ which have never had positive market share in any other configuration.

In short, the smart home appliance evolves from a networked complement to the smartphone into a networked substitute for it.

Note that violating Assumption 2 means that Lemma 2 may no longer hold, which would lead to a positive market share for $\{\varnothing, \varnothing\}$ (a Consumer with a feature phone and no smart home appliance). In either case, Proposition 4 continues to hold, and adding phone-like features to the smart speaker reduces mixed-product bundling.

\section{Discussion}

We model the evolution of the market for smartphones and complementary goods in four distinct eras. Each era is discussed in turn.

In the first era, smartphones exist as stand-alone products. They exhibit cross-side network effects but otherwise behave in a manner that is disconnected from other networked markets. A universe of complementary goods emerged providing docks, cases, chargers, vehicle interfaces, and so on, but these goods did not exhibit the kinds of cross-side network effects that would significantly affect adoption of one smartphone over another.

In the second era, firms introduce a new networked good that might provide minimal functionality on its own, but only functions fully - and indeed enjoys complementarity - when paired with a smartphone from the same vendor. For example, an early generation Apple Watch was not capable of much without a constant connection to an iPhone.

The market outcomes for strong-taste items (i.e., wearables) and weak-taste items (i.e., smart home appliances) are somewhat different, but the differences appear to be more in degree than in kind. Note that our model predicts that smartwatches are unlikely to achieve full adoption by consumers, but that smart home appliances should be expected to eventually.

In the third era, the newer networked good achieves reasonable stand-alone functionality. An example of an era 3 device is a smartwatch that does not require pairing with a smartphone. For the first time, a consumer who prefers devices from different vendors can expect each to work properly, although the complementarity between devices from the same vendor still exists.

At this point, the new networked goods theoretically pose a substitution threat to the incumbent smartphone platforms. We show that the threat from wearables like smartwatches is hypothetical, but the threat from smart home appliances is plausible given the historical trends in industries where technical capability grows faster than consumers' collective ability to harness that capability [8]. The newer device becomes good enough for most consumers, and the incumbent device withers.

In the fourth era, assuming that smart homes don't make smartphones obsolete, we posit that vendors would import the look and feel of their incumbent platform to their newer non-smartphone devices. Examples might be adding a large collection of gesture controls to smartwatch screens, or adding a home screen style interface to smart speakers.

The impact of phone-like features is quite different for each class of devices. The devices like smartwatches and other wearables with strong consumer tastes increase the utility for core customers but actually causes defections from the platform on the margins. On the other hand, devices with weak consumer tastes like smart speakers benefit from this type of stylistic integration even if does not improve the functional complementarity between the smart speaker and the smartphone.

Firms would be well served to increase the functional complementarity ("synergy") between any of their networked devices (represented by $S$ in the model). A higher $S$ leads to more consumers preferring a singlevendor bundle, increasing utility for core and marginal consumers alike.

Firms should be cautious about increasing the taste compatibility ("homogenizing the look and feel") between smartphones and devices that have stronger consumer tastes (represented by $\alpha$ in the model). We expect that wearables will follow a path toward high differentiation while smart home appliances will follow a path toward modest differentiation, but it is conceivable that either or both predictions may prove inaccurate. 


\section{Conclusion}

The conventional wisdom in economics and strategy that any two products are either complements or substitutes. In this research centered in technology markets, we identified cases in which a device, such as a smart home appliance, is a networked complement initially, but as its capabilities evolve over time, it may become a networked substitute to today's dominant computing platform, the smartphone. We identify and characterize four eras to capture this evolution of smart devices.

The second contribution of this research is that we characterize two very different emerging tech trends (wearables and smart speakers) in one parsimonious analytical framework.

Our third contribution is an economic model of the Internet of Things (IoT). As we discussed in the background, there is an extensive literature on the technical aspects of IoT, but very little understanding of the economics of IoT. We hope that this will become a growing area of research in information systems and related fields.

The fourth contribution of this work is that we analyzed the strategic impact of emerging tech trends such as wearables (smartwatches) and AI-enabled connected devices (e.g., smart speakers) on mobile computing (smartphones). As technology and other companies position themselves for the future waves of emerging tech innovations, our analysis provides managers with important actionable strategic insights:

First, the current generation of smartwatches drives sales primarily through complementarity with smartphones, but not all consumers find this combination compelling. For example, someone may prefer the iPhone to an Android phone, yet simultaneously prefer an Android Wear watch to the Apple Watch. Tying smartwatches to smartphones in this way therefore generates some deadweight loss. Stand-alone smartwatches hold the promise of serving many of these consumers.

Second, note that the ability of a smartwatch to make phone calls is not dangerous because making phone calls is a hygiene factor on a smartphone rather than a differentiating one.

Third, technology vendors would do well to increase the complementarity between their phones and watches, but the two products should remain distinct.

Fourth, on the other hand, vendors for generalpurpose smart home appliances like smart speakers do not face a similar penalty for importing smartphone features. In fact, the logical end-point is for full integration with smartphones. There is one caveat however: a household may contain members who prefer different smartphones.
The future looks very interesting for the consumers, developers, and vendors of computing platforms. Our examination of the relationship between wearables, mobile computing, and smart homes shows that these relationships are not static. In particular, if smart appliances become common in homes, vehicles, places of business, etc. then the smartphone - today's essential must-have device - may find itself suddenly redundant.

\section{References}

[1] Amelio, A. and Jullien, B., "Tying and Freebies in Twosided Markets," International Journal of Industrial Organization, 30 (5), 2007, pp. 436-446.

[2] Bakos, Y. and Brynjolfsson, E., "Bundling Information Goods: Pricing, Profits and Efficiency," Management Science, 45 (12), 1999, pp. 1613-1630.

[3] Bakos, Y. and Katsamakas, E., "Design and ownership of two-sided networks: Implications for Internet platforms," Journal of Management Information Systems, 25 (2), 2008, pp. 171-202.

[4] Cai, G., Dai, Y., and Zhou, S., "Exclusive Channels and Revenue Sharing in a Complementary Goods Market," Marketing Science, 31 (1), 2012, pp. 172-187.

[5] Carlton, D. W. and Waldman, M., "The Strategic Use of Tying to Preserve and Create Market Power in Evolving Industries," RAND Journal of Economics, 33 (2), 2002, pp. 194-220.

[6] Choi, J., "Tying in two-sided markets with multihoming," The Journal of Industrial Economics, 58 (3), 2010, pp. 607-626.

[7] Choi, J. and Jeon, D., "A Leverage Theory of Tying in Two-Sided Markets," CEPR Discussion Paper DP11484, 2016, https://ssrn.com/abstract=2834821.

[8] Christensen, C. The Innovator's Dilemma: The Revolutionary Book That Will Change the Way You Do Business. HarperBusiness Essentials, New York, 2003.

[9] Doganoglu, T., and Wright, J., "Exclusive Dealing with Network Effects," International Journal of Industrial Organization, 28 (2), 2006, pp. 145-154.

[10] Economides, N., "The economics of networks." International Journal of Industrial Organization 14(6), 1996, pp. 673-699.

[11] Economides N. and Katsamakas E., "Two-sided competition of proprietary vs. open source technology platforms.” Management Science, 52(7), 2006, pp. 10571071 . 
[12] Gans, J., "Remedies for Tying in Computer Applications," International Journal of Industrial Organization, 29 (5), 2011, pp. 505-512.

[13] Ghose, A., Tap: Unlocking the Mobile Economy. MIT Press, Cambridge, MA, 2017.

[14] Hermalin, B. and Katz, M., "Product Differentiation through Exclusivity: Is There a One-Market-Power-Rent Theorem?" working paper, 2011.

[15] Heitkoetter, H., Hildebrand, K., \& Usener, C., "Mobile Platforms as Two-Sided Markets," Proceedings of the 18th Americas Conference on Information Systems, Seattle, WA, 2012.

[16] Parker, G. and Van Alstyne, M., "Two-Sided Network Effects: A Theory of Information Product Design,"

Management Science, 51 (10), 2005, pp. 1494-1504.
[17] Rochet, J., \& Tirole, J., "Platform competition in twosided markets," Journal of the European Economic Association, 1 (4), 2003, pp. 990-1029.

[18] Rochet, J., \& Tirole, J., "Two-Sided Markets: A Progress Report," RAND Journal of Economics, 37 (3), 2006, pp. 645-667.

[19] Whinston, M., "Tying, Foreclosure, and Exclusion," American Economic Review, 80, 1990, pp. 837-859.

[20] Whitmore, A., Agarwal, A. and Da Xu, L., "The Internet of Things-A survey of topics and trends." Information Systems Frontiers, 17 (2), 2015, pp.261-274. 